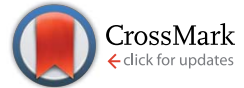

Cite this: J. Mater. Chem. A, 2014, 2 , 15972

Received 22nd April 2014

Accepted 28th July 2014

DOI: 10.1039/c4ta01995d

www.rsc.org/MaterialsA

\title{
Molecular gelation of ionic liquid-sulfolane mixtures, a solid electrolyte for high performance dye-sensitized solar cells
}

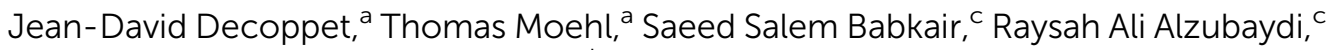

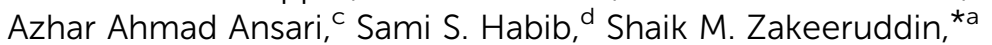 \\ Hans-Werner Schmidt ${ }^{\star e}$ and Michael Grätzel ${ }^{\star a b}$
}

\begin{abstract}
Cyclohexanecarboxylic acid-[4-(3-tetradecylureido)phenyl]amide is an efficient gelator to solidify ionic liquid electrolytes. In this paper we apply this low molecular weight gelator to solidify the newly prepared sulfolane based ionic liquid electrolyte. This solid electrolyte is successfully applied as an electrolyte for dye sensitized solar cells. This solid electrolyte is thermo-reversible, upon heating it will become a liquid and at room temperature it will solidify, facilitating the cell filling by the electrolyte. Applying this solid electrolyte we obtained $7.8 \%$ power conversion efficiency under simulated AM 1.5 full sunlight intensity. The devices with liquid and solid electrolytes were analysed by electrochemical impedance spectroscopy to explain the differences in the photovoltaic performance. These cells were also measured under outdoor conditions at Jeddah, Saudi Arabia to explore the feasibility of practical applications of this electrolyte.
\end{abstract}

\section{Introduction}

Dye sensitized solar cells (DSCs) have attracted wide spread attention in recent years due to their low cost and high efficiency ${ }^{1}$ and are presently being employed in commercial products such as light-weight flexible devices for powering portable electronic devices and as electric power producing glass panels in building integrated photovoltaic devices. The redox electrolyte plays a key role in the solar light energy conversion process shuttling electric charges from the sensitizer to the counter-electrode. All embodiments that are presently used on the commercial scale employ the triiodide/iodide $\left(\mathrm{I}_{3}{ }^{-} / \mathrm{I}^{-}\right)$couple as a charge carrier. Various types of electrolytes have been developed employing low viscosity solvents for top-level performance or non-volatile systems based on ionic liquids for high durability. In the latter case mass transport limitations of the photocurrent are frequently encountered depending on the viscosity of the liquid employed. On the

${ }^{a}$ Laboratory for Photonics and Interfaces, Institute of Chemical Sciences and Engineering, Swiss Federal Institute of Technology, CH-1015 Lausanne, Switzerland. E-mail: shaik.zakeer@epfl.ch; michael.graetzel@epfl.ch

${ }^{b}$ Center of Nanotechnology, King Abdulaziz University, Jeddah, 21589, Saudi Arabia ${ }^{c}$ Center of Nanotechnology, Department of Physics, Faculty of Science, King Abdulaziz University, Jeddah, 21589, Saudi Arabia

${ }^{d}$ Center of Nanotechnology, Department of Aeronautical Engineering, King Abdulaziz University, Jeddah, 21589, Saudi Arabia

${ }^{e}$ Macromolecular Chemistry I, Bayreuther Institut für Makromolekülforschung (BIMF) and Bayreuther Zentrum für Grenzfächen und Kolloide (BZKG), University of Bayreuth, D-95447, Bayreuth, Germany. E-mail: hans-werner.schmidt@ uni-bayreuth.de other hand, the loss of volatile solvents through leaks is a practical concern for volatile solvent-based cell embodiments. The reduction of volatility and fluidity of the liquid electrolyte by jellification is advantageous as it not only prevents leakage but also avoids undesirable contact with solvent sensitive cell constituents, such as the sealing material. In addition, avoiding organic solvents can reduce the risk of dye desorption from the $\mathrm{TiO}_{2}$ surface into the electrolyte. Considerable effort has been made to replace liquid with quasi-solid or solid-state electrolytes using low molecular weight compounds, polymers or oxide nanoparticles as jellifying materials. ${ }^{2-8}$ Low molecular weight gelators have attracted particular attention due to their numerous industrial applications such as in cosmetics, food processing, lubrications, etc. ${ }^{9}$ Solidification occurs upon dissolving a small quantity of the gel forming agent into a hot liquid electrolyte and subsequent cooling below the gel transition temperature. The three-dimensional networks formed in the quasi-solid gel electrolyte immobilize the liquid component to a variable extent. ${ }^{9}$ The reversibility of these quasisolid electrolytes depending on the temperature is the attracting feature compared to other quasi-solid electrolytes prepared with metal oxide nanoparticles or polymer electrolytes. Quasi-solid electrolytes prepared using a low molecular weight gelator are liquid at higher than the gel transition temperature and can fill easily the $\mathrm{TiO}_{2}$ mesopores whereas this is not possible with other nanoparticle based quasi-solid electrolytes. Here we report on the effect of gelation on the photovoltaic performance of a recently reported sulfolane based ionic liquid electrolyte, which exhibits exceptional stability under long term heat stress and light soaking. ${ }^{10}$ 


\section{Results and discussion}

We selected cyclohexanecarboxylic acid-[4-(3-tetradecylureido) phenyl]amide coded as CTP as a molecular gel forming agent in order to solidify the ionic liquid-sulfolane composite electrolyte. The molecular structure of the gelator CTP is designed to dissolve at elevated temperatures in a broad range of solvents with different polarities. The amide moiety and the urea moiety enable self-assembly upon cooling. The balance of these hydrogen-bonding units with the terminal cyclohexane group and the linear aliphatic alkyl chain (C14) enables the molecule to form nanofibrillar structures, which cause efficient gelation in a large variety of solvents. ${ }^{11-13}$ The influence of the solvent polarity and gelator concentration on the dissolution and selfassembly of gelation is concentration dependent. ${ }^{13}$ The chemical structure of CTP is shown in Scheme 1. At $2 \%$ weight the electrolyte is a liquid when heated to above $100{ }^{\circ} \mathrm{C}$ but becomes a solid upon cooling to room temperature. The diffusion coefficients of liquid and gel electrolytes were calculated by electrochemical impedance measurements by using a symmetric cell. ${ }^{21}$ The Nyquist plot of the symmetric cells at $0 \mathrm{~V}$ is shown in Fig. 1. The diffusion coefficients of triiodide in the liquid electrolyte and in the gelled electrolyte mixture were determined to be $8 \times 10^{-7}$ and $6 \times 10^{-7} \mathrm{~cm}^{2} \mathrm{~s}^{-1}$, respectively. The diffusion coefficients were also calculated by the limiting currents measured by the current-voltage characteristics of such symmetric cells. The trend for liquid and gel electrolytes is similar although the values are somewhat higher compared to the impedance measurement $\left(10 \times 10^{-7}\right.$ and $8 \times 10^{-7} \mathrm{~cm}^{2} \mathrm{~s}^{-1}$, respectively).

The newly formulated gel redox electrolyte was employed in DSCs featuring a double layer mesoporous $\mathrm{TiO}_{2}$ film a $4 \mu \mathrm{m}$

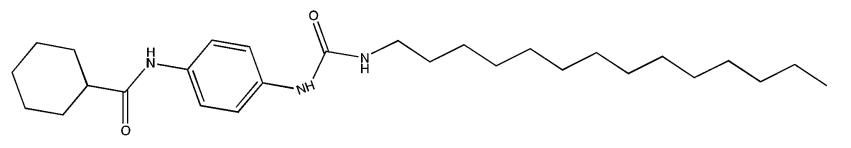

Scheme 1 Molecular structure of the gelator.

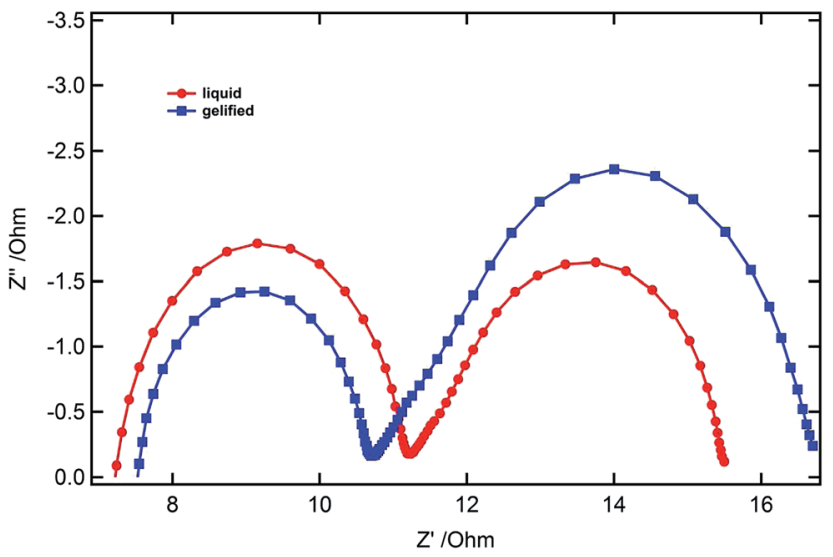

Fig. 1 Nyquist plot of the symmetric devices only with the liquid and gelled electrolytes recorded at $0 \mathrm{~V}$. light scattering layer of large $400 \mathrm{~nm}$ sized particles being superimposed on a $8 \mu \mathrm{m}$ thick nanoparticle layer. The amphiphilic ruthenium complex C106 was used as a sensitizer. Table 1 compares the open circuit voltage $\left(V_{\mathrm{oc}}\right)$, fill factor $(\mathrm{FF})$, short circuit current density $\left(J_{\mathrm{sc}}\right)$ and standard power conversion efficiency (PCE), the photovoltaic for the solid and liquid electrolyte devices. The PCE of the liquid electrolyte is a little higher than that of the gel electrolyte due to the difference in the $J_{\mathrm{sc}}$ values. Fig. 2 shows $J-V$ characteristics of the two DSCs measured under standard AM $1.5 \mathrm{G}$ illumination at $100 \mathrm{~mW}$ $\mathrm{cm}^{-2}$ while photocurrent action spectra are presented as an inset in Fig. 2. The incident photon to conversion efficiency (IPCE) covers a broad spectral range from $440 \mathrm{~nm}$ to $760 \mathrm{~nm}$, reaching its maximum of $81 \%$ at $580 \mathrm{~nm}$. The short circuit photocurrents calculated from the overlap integral of these curves with the standard global AM 1.5 solar emission spectrum agree within $4 \%$ with the measured photocurrent showing that any spectral mismatch between our solar simulator and the standard AM 1.5G solar emission is small. The long-term stability of devices with liquid electrolytes is shown to be stable when they are aged at $60{ }^{\circ} \mathrm{C}$ light soaking as reported. ${ }^{10}$ The stability of gel electrolyte devices was excellent and the relative deviation of the power conversion efficiency was within $2 \%$ of the initial performance after aging for 3 weeks under light soaking at $60{ }^{\circ} \mathrm{C}$ in a solar simulator $\left(100 \mathrm{~mW} \mathrm{~cm}{ }^{-2}\right)$.

We employed electrochemical impedance spectroscopy (EIS) measurements to examine differences in the photovoltaic characteristics of liquid and gel electrolyte based DSCs. Using the transmission line model ${ }^{18-20}$ we extracted from the Nyquist plot the key electric device parameters, i.e. the

Table 1 Photovoltaic parameters of DSCs employing liquid or solid gel electrolytes measured under standard AM 1.5G illumination at $100 \mathrm{~mW}$ $\mathrm{cm}^{-2}$. The average values of 5 devices are shown in the brackets

\begin{tabular}{lllll}
\hline Electrolyte & $J_{\mathrm{sc}}\left(\mathrm{mA} \mathrm{cm}^{-2}\right)$ & $V_{\text {oc }}(\mathrm{mV})$ & $\mathrm{FF}$ & $\eta$ \\
\hline Liquid & 16.2 & 717 & 0.72 & 8.43 \\
Gel & $15.2(15.3)$ & $717(716)$ & $0.72(0.68)$ & $7.78(7.5)$
\end{tabular}

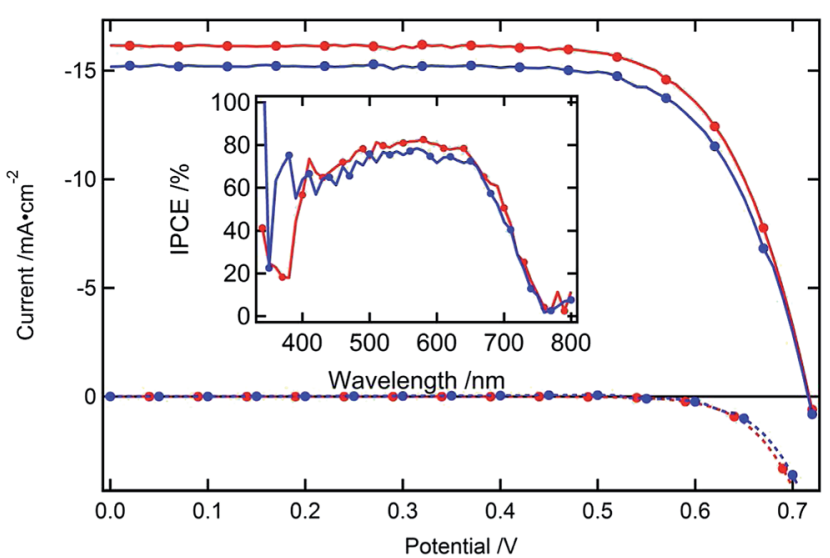

Fig. $2 \quad I-V$ characteristics of two photovoltaic cells using liquid (red) or solid gel (blue) electrolytes. The inset shows the IPCE of the devices. 
transport resistance for the electrons through the nanocrystalline $\mathrm{TiO}_{2}$ film ( $R_{\text {trans }}$ ), the recombination resistance between $\mathrm{TiO}_{2}$ conduction band electrons and triiodide ions in the electrolyte $\left(R_{\mathrm{CT}}\right)$, and the chemical capacitance of the $\mathrm{TiO}_{2}$ nanocrystals $\left(C_{\text {chem }}\right)$. Results are shown in Fig. 3 . The $R_{\mathrm{CT}}$ values determined are in good agreement with the behaviour of the dark current (see Fig. 3a and b). Since the electrolyte compositions of liquid and gel electrolytes are similar, except for the added molecular gelator, the characteristics of the photoanode are very similar though it seems that in the case of the gel electrolyte the conduction band is slightly shifted upwards ( 10 to $15 \mathrm{mV}$ ). On the other hand the recombination rate is increased and the transport seems also a bit slower rendering charge collection less efficient compared to the liquid electrolyte. Nevertheless all the observed differences are small and the conduction band move will be compensated by the faster recombination, which is responsible for obtaining similar $V_{\text {oc }}$ values for the two devices (see Fig. 3d). In the case of the resistances contributing to the overall series resistance of the devices ( $R_{\text {series }}$ (from cables, contacts and the electrolyte resistance), $R_{\mathrm{CE}}$ (from the charge transfer at the counter electrode) and $R_{\text {Dif }}$ (from the diffusion of the triiodide in the electrolyte)) the characteristics of the devices are very close, though the ones with a quasi-solid electrolyte show a higher $R_{\mathrm{CE}}$ and a higher $R_{\text {Dif }}$ (see Fig. 3c). The small difference in the photovoltaic performance of liquid and gel electrolyte devices arises from the $J_{\mathrm{sc}}$ value. The lower $J_{\mathrm{sc}}$ of the gel electrolyte device may be attributed to its larger Warburg impedance indicating an impediment of triiodide diffusion through the network of the gel. Alternatively the higher conduction band position of the $\mathrm{TiO}_{2}$ in the gel based DSCs may reduce the yield of electron injection of electrons from the excited dye into the $\mathrm{TiO}_{2}$ conduction band, which is supported by the IPCE spectra.

Outdoor measurements were carried out in Jeddah Saudi Arabia with a DSC employing the $\mathrm{C} 106$ ruthenium complex as a sensitizer, the Z988 electrolyte and 2\% CTP gelator. Data are shown in Fig. 4. Measurements were carried out on January 2, 2014 from 8 a.m. to 5 p.m. The active area of the cell was a round spot of $6 \mathrm{~mm}$ diameter. A black mask with a round aperture having a diameter of $8.5 \mathrm{~mm}$ was employed for all the measurements to avoid inflation of photocurrents from light piping by uncovered glass. Details of the outdoor conditions are shown in Table 2 along with the measured key photovoltaic performance parameters. Impressively, the PCE of the cells was close to $9 \%$ around noontime when the temperature reached nearly $37^{\circ} \mathrm{C}$ confirming the excellent performance of the new electrolyte formulation under the hot conditions prevailing in Jeddah even in the beginning of January. The laboratory results taken from 5 cells showed the same trend as measured under outdoor conditions. The $V_{\mathrm{oc}}$ varied by less than $5 \mathrm{mV}$ during the day, along with small changes in the fill factor and the maximum power point voltage. This has practical advantages as it obviates the need for using a power tracking system to obtain the maximal output. By contrast the $J_{\mathrm{sc}}$ was more strongly affected by the AM number and the hour of the day due to variations in the intensity and spectral distribution of the incident sunlight. Importantly the PCE varied over a much smaller range than the photocurrent remaining between 8 and $9 \%$ during most of the day except for the first and the last measurement where the AM number was over 4. Due to the long path through the atmosphere the solar emission shifts here to the red and near IR reducing its spectral overlap with the sensitizer and hence the photocurrent.
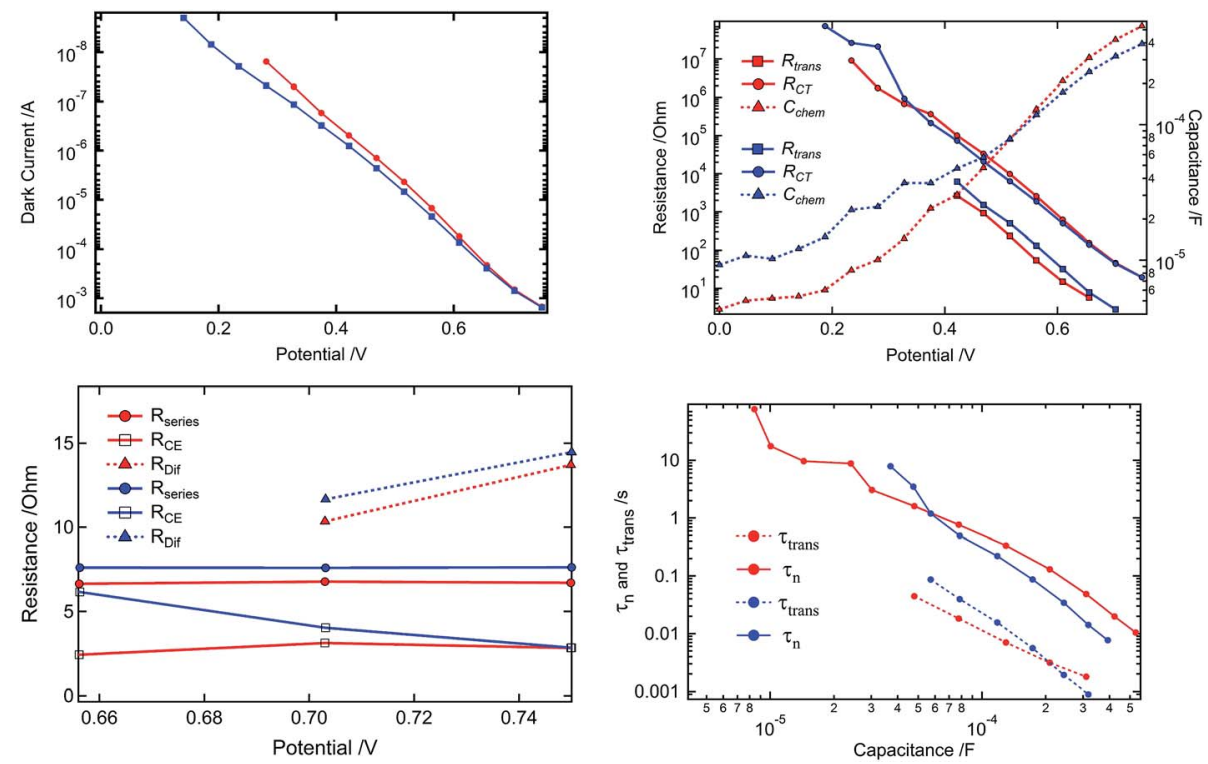

Fig. 3 (a) Dark current of the two different devices (red: Z988; blue: Z988 with gel). (b) Main parameters extracted from EIS measurements. Red points for the $Z 988$ electrolyte and blue points for the Z988 with the CTP gel forming agent. (c) Series resistance contribution for the different devices with the resistance from the cables and contacts $\left(R_{\text {series }}\right)$, the counter electrode resistance $\left(R_{\mathrm{CE}}\right)$ and the diffusion resistance for the $\mathrm{I}_{3}{ }^{-}$in the electrolyte $\left(R_{\mathrm{DIF}}\right)$. (d) Electron lifetime and transport time plotted against the chemical capacitance. 

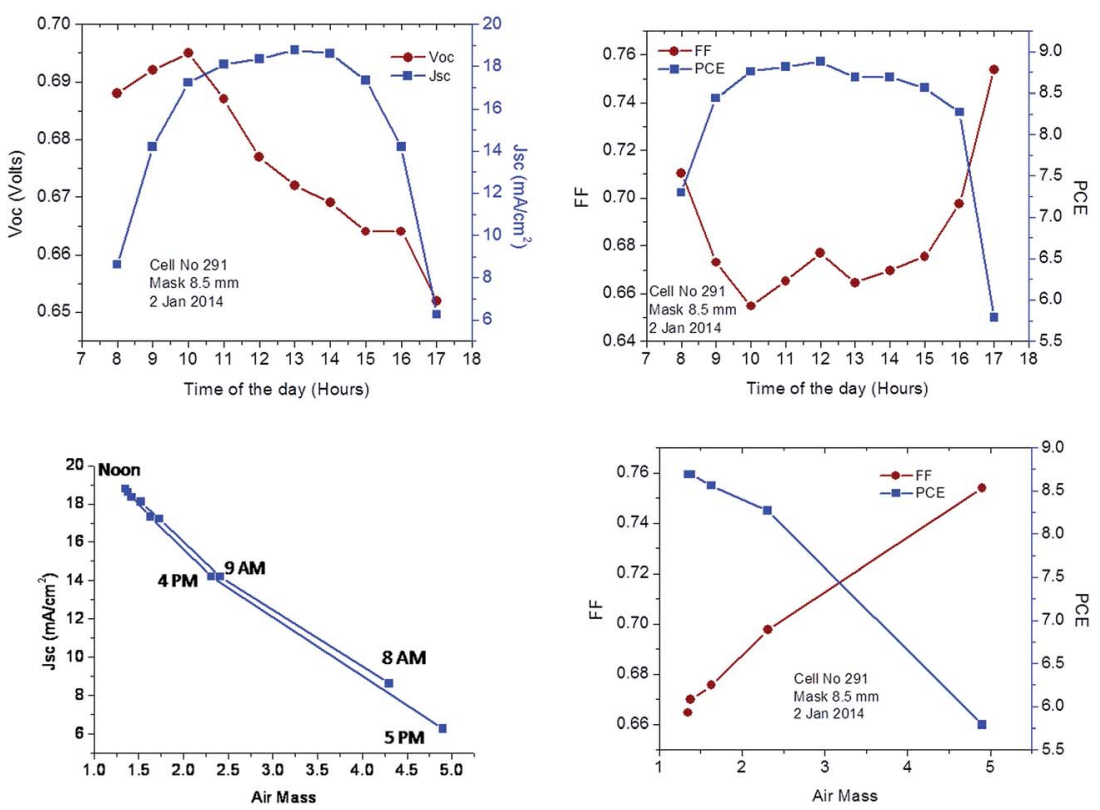

Fig. 4 Outdoor photovoltaic performance in Jeddah Saudi Arabia of cell DSC-291 employing the C106 ruthenium complex as a sensitizer, Z988 electrolyte and 2\% CTP gelator. Measurements were carried out on January 2, 2014 from 8 a.m. to 5 p.m. The active area of the cell was a round spot of $6 \mathrm{~mm}$ diameter. A black mask with an aperture diameter of $8.5 \mathrm{~mm}$ was employed for all the measurements. Top left: the $V_{\mathrm{oc}}$ and $J_{\mathrm{sc}}$ are plotted as a function of the time during the day. Top right: the FF and PCE are plotted as a function of the time during the day. Bottom left: the $J_{\text {sc }}$ is plotted against the air mass number. Bottom right: the FF and PCE are plotted against the air mass number.

Table 2 List of outdoor conditions and key photovoltaic performance parameters for a cell measured in Jeddah Saudi Arabia with a DSC employing the $\mathrm{C} 106$ ruthenium complex as a sensitizer, the $Z 988$ electrolyte and $2 \%$ CTP gelator. $P_{\text {in }}=$ incident power intensity; $J_{\text {sc }}=$ short circuit current density; $V_{M P}=$ photovoltage at the maximum output power point; $J_{M P}=$ current density at the maximum output power point; and $\mathrm{MP}=$ the maximum output power point

\begin{tabular}{|c|c|c|c|c|c|c|c|c|c|c|}
\hline Time & $\begin{array}{l}\text { Temp. } \\
\left({ }^{\circ} \mathrm{C}\right)\end{array}$ & $\mathrm{AM}$ & $\begin{array}{l}P_{\text {in }} \\
\left(\mathrm{mW} \mathrm{cm}^{-2}\right)\end{array}$ & $V_{\mathrm{oc}}(\mathrm{V})$ & $\begin{array}{l}J_{\mathrm{sc}} \\
\left(\mathrm{mA} \mathrm{cm}^{-2}\right)\end{array}$ & $V_{\mathrm{MP}}(\mathrm{V})$ & $\begin{array}{l}J_{\mathrm{MP}} \\
\left(\mathrm{mA} \mathrm{cm}{ }^{-2}\right)\end{array}$ & $\begin{array}{l}P_{\text {out }} \\
\left(\mathrm{mW} \mathrm{cm}{ }^{-2}\right)\end{array}$ & $\mathrm{FF}$ & PCE \\
\hline 8 a.m. & 22.5 & 4.30 & 57.8 & 0.688 & 8.630 & 0.547 & 7.711 & 4.218 & 0.710 & 7.30 \\
\hline 10 a.m. & 28.5 & 1.73 & 89.52 & 0.695 & 17.22 & 0.498 & 15.74 & 7.84 & 0.655 & 8.76 \\
\hline 11 a.m. & 32.5 & 1.52 & 92.78 & 0.687 & 18.11 & 0.511 & 16.20 & 8.28 & 0.665 & 8.82 \\
\hline Noon & 36.6 & 1.42 & 94.68 & 0.677 & 18.36 & 0.516 & 16.30 & 8.41 & 0.677 & 8.88 \\
\hline 3 p.m. & 35.0 & 1.63 & 90.92 & 0.664 & 17.33 & 0.516 & 15.07 & 7.77 & 0.676 & 8.56 \\
\hline 4 p.m. & 32.8 & 2.31 & 79.66 & 0.664 & 14.22 & 0.513 & 12.84 & 6.59 & 0.698 & 8.27 \\
\hline 5 p.m. & 30.0 & 4.90 & 53.2 & 0.652 & 6.260 & 0.537 & 5.739 & 3.08 & 0.754 & 5.79 \\
\hline
\end{tabular}

\section{Summary and conclusions}

We show that the molecular gelator CTP is very effective in solidifying the high-endurance electrolyte Z-988 composed of a mixture of sulfolane with an ionic liquid at levels as low as $2 \%$. Solidification has only a small impact on the key photovoltaic performance parameters. The open circuit voltage $\left(V_{\mathrm{oc}}\right)$ and fill factor $(\mathrm{FF})$ remain practically unaffected while the photocurrent drops due to an increase in the Warburg diffusion resistance for triiodide ions in the presence of CTP. The first outdoor experiments performed in Jeddah Saudi Arabia gave very promising results. Importantly power conversion efficiencies exceed those measured under standard AM 1.5 conditions, the maximum PCE of $8.9 \%$ being reached at around noontime when the temperature reached $37^{\circ} \mathrm{C}$. The $V_{\text {oc }}$ varied by less than $5 \mathrm{mV}$ during the day, along with small changes in the fill factor and the maximum power point voltage. This has practical advantages as it obviates the need for using power tracking systems to obtain the maximal output from the PV panel. As expected at high air mass numbers the photocurrent decreased substantially due to the red shift of the solar emission spectrum. Overall the data are of great practical value and forebode well for the large-scale use of dye-sensitized solar cells in desert climates. 


\section{Experimental section}

\section{Reagents}

1,3-Dimethylimidazolium iodide (DMII) and 1-ethyl-3-methylimidazolium iodide (EMII) were purchased from Io-Li-Tec and 1-ethyl-3-methylimidazolium tetracyanoborate (EMITCB) was from Merck. C106 dye, dineohexyl bis-(3,3-dimethyl-butyl)phosphinic acid (DINHOP) and cyclohexanecarboxylic acid-[4(3-tetradecylureido)phenyl]amide (CTP) were synthesized as reported previously. ${ }^{11-13,17-19}$ Sulfolane (99\%) was purchased from Aldrich and distilled before use.

\section{Photovoltaic device fabrication}

Dye sensitized solar cells were fabricated using a double-layer photoanode made of mesoporous $\mathrm{TiO}_{2}$ as reported previously. ${ }^{20}$ Prior to use the photoanodes were briefly sintered again and after cooling to $80^{\circ} \mathrm{C}$ immersed for $16 \mathrm{~h}$ at room temperature in a $0.3 \mathrm{mM}$ C106 dye solution in 10\% DMSO and tert-butanol : acetonitrile $(1: 1 \mathrm{v} / \mathrm{v})$ with DINHOP as a co-adsorbent, the molar ratio of dye to DINHOP being $4: 1$. The electrode was then rinsed with $\mathrm{CH}_{3} \mathrm{CN}$ and dried. The stained substrates were again rinsed with $\mathrm{CH}_{3} \mathrm{CN}$ and subsequently sealed with pieces of thermally platinized (a drop of $8 \mathrm{mM}$ hexachloroplatinic solution in 2-propanol, heated to $425{ }^{\circ} \mathrm{C}$ ) FTO glass (TEC15, Pilkington), which served as a counter electrode. $25 \mu \mathrm{m}$ thick Surlyn (Dupont) was used as a binder and a spacer. The electrolytes were introduced to the cells via pre-drilled holes in the counter electrodes. The composition of the $\mathrm{Z952}$ electrolyte is as follows: DMII/EMII/EMITCB/ $/ \mathrm{I}_{2} / \mathrm{NBB} / \mathrm{GNCS}$ (molar ratio $12: 12: 16: 1.67: 3.33: 0.67)$. The final liquid electrolyte (labelled as Z988) was prepared by adding $50 \%$ of sulfolane $(\mathrm{v} / \mathrm{v})$. The gel electrolyte was prepared by adding $2 \mathrm{wt} \%$ of cyclohexanecarboxylic acid-[4-(3-tetradecylureido)phenyl]amide gelator to the liquid electrolyte and warming, after cooling down to room temperature the electrolyte became solid.

\section{Electrochemical impedance spectroscopy (EIS) measurements}

EIS measurements were performed with a PGSTAT30 frequency analyzer from Autolab (Eco Chemie B.V., Utrecht, The Netherlands) together with the Frequency Response Analyzer module providing voltage modulation in the desired frequency range. The Z-view software (v2.8b, Scribner Associates Inc.) was used to analyze the impedance data. The EIS experiments were performed at a constant temperature of $20{ }^{\circ} \mathrm{C}$ in the dark. The diffusion coefficients of liquid and gel electrolytes were calculated by measuring a symmetric cell (dummy cell) consisting only of platinized FTO electrodes on both sides separated by $25 \mu \mathrm{m}$ thick Surlyn (Dupont).

$$
D_{\mathrm{I}^{-}}=\frac{J_{\lim } l}{2 n e c N_{\mathrm{av}}}
$$

The impedance measurement of the dummy devices for the determination of the diffusion coefficient of $\mathrm{I}_{3}{ }^{-}\left(D_{\mathrm{I}_{3}{ }^{-}}\right)$was done at $0 \mathrm{~V}$ and the data analysed on the basis of the Randles circuit which includes a series resistance, a charge transfer resistance, the double layer capacitance and the element used to extract the diffusion coefficient, the Warburg diffusion element. Applying this equivalent circuit to the impedance spectra yields the Warburg parameter. After the fitting procedure the diffusion coefficient $D_{\mathrm{I}_{3}{ }^{-}}$is calculated by the relationship $D_{\mathrm{I}_{3}{ }^{-}}=\delta^{2} / \mathrm{W}$ where $\delta$ is half of the distance between the two Pt electrodes and $W$ the Warburg coefficient extracted from the analysis of the Nyquist plot ( $20 \mu \mathrm{m}$ was used instead of the $25 \mu \mathrm{m}$ since due to the hot melt process the thickness is reduced). ${ }^{21}$

The measurements for the determination of $D_{\mathrm{I}_{3}}$ - by cyclic voltammetry were performed in a potential range of $-0.9 \mathrm{~V}$ to $+0.9 \mathrm{~V}$. The limiting current density $\left(J_{\text {lim }}\right)$ is obtained by dividing the limiting current $\left(I_{\lim }\right)$ by the surface exposed to the electrolyte. The diffusion coefficient is calculated by using the above-mentioned formula, where $l$ is the distance between the two electrodes, $n$ is the number of exchanged electrons, $e$ is the elementary charge, $c$ is the triiodide concentration and $N_{\mathrm{av}}$ is the Avogadro number. ${ }^{21}$

The impedance spectra of the DSC devices were recorded at potentials varying from $0 \mathrm{~V}$ to $V_{\mathrm{oc}}$ at frequencies ranging from $1 \mathrm{MHz}$ to $0.1 \mathrm{~Hz}$, with a sinusoidal potential pertubation of $10 \mathrm{mV}$. The photoanode $\left(\mathrm{TiO}_{2}\right)$ was used as the working electrode and the Pt counter electrode (CE) was used as both the auxiliary electrode and the reference electrode. These obtained spectra were fitted with the transmission line model. ${ }^{\mathbf{1 8 - 2 0}}$.

\section{Acknowledgements}

Financial and technical support of this work by King Abdulaziz University (KAU) Jeddah, Saudi Arabia under grant number $1-20-1432 / \mathrm{HiCi}$ is gratefully acknowledged. M.G. thanks the European Research Council for an Advanced Research Grant (ARG 247404) funded under his "Mesolight" project. H.-W.S. gratefully acknowledges the Bavarian State Ministry of Science, Research, and the Arts for the financial support by the Collaborative Research Network "Solar Technologies go Hybrid".

\section{References}

1 B. O'Regan and M. Grätzel, Nature, 1991, 353, 737-740.

2 A. F. Nogueira, J. R. Durrant and M. A. De Paoli, Adv. Mater., 2001, 13, 826-830.

3 I. Chung, B. Lee, J. He, R. P. H. Chang and M. G. Kanatzidis, Nature, 2012, 485, 486-489.

4 P. Wang, S. M. Zakeeruddin, I. Exnar and M. Grätzel, Chem. Commun., 2002, 2972-2973.

5 W. Kubo, S. Kambe, S. Nakade, T. Kitamura, K. Hanabusa, Y. Wada and S. Yanagida, J. Phys. Chem. B, 2003, 107, 4374-4381.

6 T. Stergiopoulos, E. Rozi, R. Hahn, P. Schmuki and P. Falaras, Adv. Energy Mater., 2011, 1, 569-572.

7 P. Wang, S. M. Zakeeruddin and M. Grätzel, J. Fluorine Chem., 2004, 125, 1241-1245.

8 P. Wang, S. M. Zakeeruddin, P. Comte, I. Exnar and M. Graetzel, J. Am. Chem. Soc., 2003, 125, 1166-1167.

9 P. Terech and R. G. Weiss, Chem. Rev., 1997, 97, 3133-3159. 
10 M. Marszalek, F. Duriaux-Arendse, J.-D. Decoppet, S.-S. Babkair, A.-A. Ansari, S. S. Habib, M. Wang, S. M. Zakeeruddin and M. Grätzel, Adv. Energy Mater., 2014, 4, 1301235.

11 N. Mohmeyer and H.-W. Schmidt, Chem.-Eur. J., 2005, 11(3), 863-872.

12 N. Mohmeyer, F. D. Kuan, P. Wang, H.-W. Schmidt, S. M. Zakeeruddin and M. Graetzel, J. Mater. Chem., 2006, 16(29), 2978-2983.

13 N. Mohmeyer and H.-W. Schmidt, Chem.-Eur. J., 2007, 13(6), 4499-4509.

14 Y. Cao, Y. Bai, Q. Yu, Y. Cheng, S. Liu, D. Shi, F. Gao and P. Wang, J. Phys. Chem. C, 2009, 113, 6290-6297.

15 M. Wang, S. Plogmaker, R. Humphry-Baker, P. Pechy, H. Rensmo, S. Zakeeruddin and M. Grätzel, ChemSusChem, 2012, 5, 181-187.
16 M. Wang, X. Li, H. Lin, P. Pechy, S. M. Zakeeruddin and M. Grätzel, Dalton Trans., 2009, 10015-10020.

17 P. Wang, S. Zakeeruddin, P. Comte, R. Charvet, R. HumphryBaker and M. Grätzel, J. Phys. Chem. B, 2003, 107, 1433614341.

18 F. Fabregat-Santiago, J. Bisquert, G. Garcia-Belmonte, G. Boschloo and A. Hagfeldt, Sol. Energy Mater. Sol. Cells, 2005, 87, 117-131.

19 F. Fabregat-Santiago, G. Garcia-Belmonte, I. Mora-Sero and J. Bisquert, Phys. Chem. Chem. Phys., 2011, 13, 9083-9118.

20 M. Wang, C. Grätzel, S. Moon, R. Humphry-Baker, N. Rossier-Iten, S. M. Zakeeruddin and M. Grätzel, Adv. Funct. Mater., 2009, 19, 2163-2172.

21 A. Hauch and A. Georg, Electrochim.Acta, 2001, 46, 34573466. 